2009-6

\title{
Dynamics and Control of Cable-Drogue System in Aerial Recovery of Micro Air Vehicles Based on Gauss's Principle
}

\author{
Liang Sun \\ Brigham Young University - Provo, solbuaa@gmail.com \\ Randal W. Beard \\ Brigham Young University - Provo, beard@byu.edu \\ Mark B. Colton \\ Brigham Young University - Provo, colton@byu.edu \\ Timothy W. McLain \\ Brigham Young University - Provo, mclain@byu.edu \\ Follow this and additional works at: https://scholarsarchive.byu.edu/facpub \\ Part of the Electrical and Computer Engineering Commons, and the Mechanical Engineering \\ Commons
}

\section{Original Publication Citation}

Sun, L., Beard, R., Colton, M., and McLain, T. Dynamics and Control of Cable-Drogue System in Aerial Recovery of Micro Air Vehicles Based on Gauss's Principle. Proceedings of the 2009 American Control Conference, pp. 4729-4734, June 2009, St. Louis, Missouri.

\section{BYU ScholarsArchive Citation}

Sun, Liang; Beard, Randal W.; Colton, Mark B.; and McLain, Timothy W., "Dynamics and Control of CableDrogue System in Aerial Recovery of Micro Air Vehicles Based on Gauss's Principle" (2009). Faculty Publications. 1538.

https://scholarsarchive.byu.edu/facpub/1538

This Peer-Reviewed Article is brought to you for free and open access by BYU ScholarsArchive. It has been accepted for inclusion in Faculty Publications by an authorized administrator of BYU ScholarsArchive. For more information, please contact ellen_amatangelo@byu.edu. 


\title{
Dynamics and Control of Cable-Drogue System in Aerial Recovery of Micro Air Vehicles Based on Gauss's Principle
}

\author{
Liang Sun, Randal W. Beard, Mark B. Colton, Timothy W. McLain
}

\begin{abstract}
This paper presents a new concept for aerial recovery of Micro Air Vehicles (ARMAVs) using a large mothership and a recovery drogue. The mothership drags a drogue attached to a cable and the drogue is controlled to match the flight patten of the MAV. This paper uses Gauss's Principle to derive the dynamic model of the cable-drogue systems. A controllable drogue plays a key role in recovering MAVs in windy conditions. We develop a control approach for the drogue using its drag coefficient. Simulation results based on multilink cable-drogue systems present the feasibility of the aerial recovery concept and the controllability of the drogue.
\end{abstract}

\section{INTRODUCTION}

Unmanned Aircraft Systems (UAS) have become an essential tool for warfighters. While high-altitude, long-endurance UASs like the Predator and the Global Hawk provide persistent intelligence, surveillance, and reconnaissance (ISR) capabilities, they are a scarce resource that cannot be given specific data-gathering tasks by individual troops. At the other end of the spectrum are backpackable Micro Air Vehicles (MAVs), with wingspans of less than 15 inches, that theoretically can be carried by every warfighter.

Backpackable MAVs enable warfighters on the ground to gather time-critical, over-the-hill ISR information. However retrieving the MAV is problematic because landing the vehicle near the soldier could disclose his/her location to an enemy. Another potential application of MAVs is collecting battle damage information. Again for this application, retrieval of the MAV after it has performed its mission is difficult because target locations are often deep in enemy territory, and the MAV may not have enough fuel to return home.

The relatively low cost of MAVs suggests that they may be expendable; thereby removing the need for recovery. However, even if the costs are low, MAVs still contain critical and often classified technology which needs to be kept out of enemy hands. One option is to destroy the MAV or damage the electronics so that it cannot be reused or reverse engineered. However, most of the solutions that have been proposed require additional payload on the MAV. Cost considerations and the potential that MAV technology could fall into enemy hands will limit the use of this technology.

Liang Sun, Ph.D student in Department of Electrical and Computer Engineering, Brigham Young University, Provo, UT 84602, USA sun.1iang@byu.edu

Randal W. Beard, Professor in Department of Electrical and Computer Engineering, Brigham Young University, Provo, UT 84602, USA beard@byu.edu

Mark B. Colton and Timothy W. McLain, Professors in Department of Mechanical Engineering, Brigham Young University, Provo, UT 84602, USAcolton@byu.edu mclain@byu.edu
Innovative recovery techniques are therefore critical to ubiquitous use of MAV technology. The primary difficultly with aerial recovery is the relative size and speed of the mothership compared to the MAV. Aerial recovery is much like aerial refueling where the goal is to extend the operational lifetime of the asset. However, in aerial refueling, the fighter jet and the tanker can match their airspeeds, which is not possible with MAVs and larger aircraft. One potential solution to this problem is to use helicopters for the recovery operation. However, helicopters produce significant prop wash making it difficult for the MAV to operate in its vicinity.

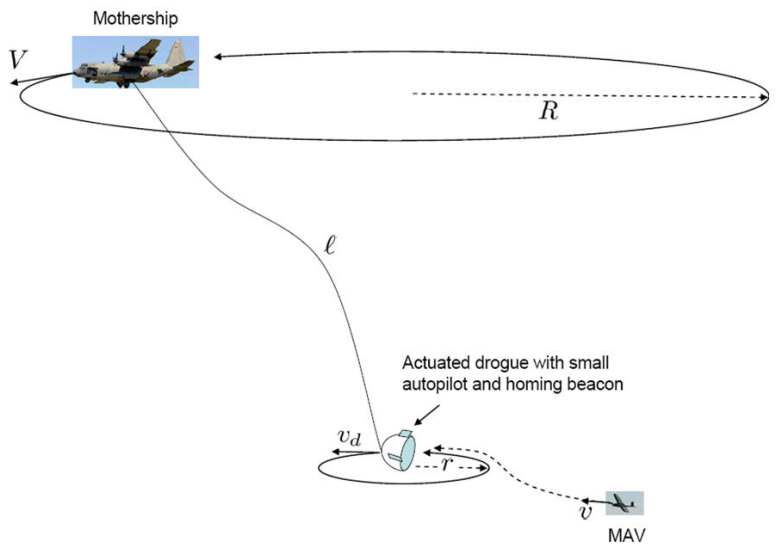

Fig. 1. This figure shows the baseline concept described in the paper. The mothership recovers a MAV by towing a long cable attached to a drogue. The drogue is actuated and can maneuver and communicate with the MAV to facilitate successful capture. The MAV uses missile guidance strategies to intercept the drogue.

Our approach is motivated by recent advances on the dynamics of towed cable systems, where a towplane drags a cable with a drogue at the end. In early work on this problem, [1] shows that if the towplane is in a constantangular-rate orbit of radius $\mathrm{R}$, and the drogue has sufficient aerodynamic drag, then the motion of the drogue has a stable orbit of radius $r \ll R$. Since the angular rates of the towplane and the drogue are identical, the speed of the drogue will be significantly less than the speed of the towplane. Reference [2] shows that the towplane-cabledrogue system is differentially flat with the position of the drogue being the flat output. In essence, this means that the trajectory of the towplane is uniquely prescribed by the motion of the drogue. Reference [2] uses the differential flatness property to design a path planning algorithm for the 
towplane with the objective of minimizing the orbit radius of the drogue. In more recent work, [3] and [4] give a detailed description of the dynamics of circularly towed drogues and design strategies for moving from one orbit configuration to another. The objective in [3] and [4] is precision pickup and delivery of payloads on the ground by a fixed-wing aircraft. Therefore, the focus in [2] [3] [4], is on minimizing the orbit radius of the drogue.

For aerial recovery of MAVs, we take a slightly different approach to the problem. As shown in Figure 1, rather than attempting to minimize the radius of the orbit of the drogue, our objective will be to place the drogue in a stable orbit whose radius $r$ is greater than the minimum turning radius of the MAV. We note that allowing the drogue to have a larger radius significantly simplifies the challenges [3] [4]. The basic idea is to maneuver the towplane, or mothership, so that the drogue enters a stable orbit at an airspeed that is slightly below the nominal airspeed of the MAV. The MAV will then be maneuvered to enter the same orbit at its nominal airspeed and will therefore overtake the drogue with a relatively slow closing speed.

In this paper, we focus on modeling the multi-link cabledrogue dynamics using Gauss's Principle. We also derive a potential control strategy for the drogue using a controllable aerodynamic drag coefficient. Simulations illustrate the aerial recovery concept.

\section{Dynamics of CABle-Drogue Systems}

The mathematical model of the cable-drogue or towedcable systems is well established in the literature for both air and underwater environments [2] [3] [5] [6] [7]. As is typical, we will use a discretized finite element or lumped mass representation of the cable dynamics.

Using the approaches in [2] [3] [5] [6] [7], the internal and external forces need to be described explicitly. Reference [2] develops towed-cable dynamics based on the Lagrange approach which requires an explicit derivation of the tension in the cable. Reference [3] derives the equations of the motion by introducing cable attitude angles. Unfortunately, both of these approaches generate complicated equations for the system dynamics model.

As an alternative, we develop the mathematical model of the cable-drogue systems based on the work of Udwadia and Kalaba [8]. A similar method was used in the context of path planning in [9]. The method is especially wellsuited to problems with complex internal forces. Rather than computing the force directly, the kinematic constraints are employed.

\section{A. Gauss's Principle}

Consider a system of $n$ particles with mass $m_{1}, m_{2}, \ldots, m_{n}$. Let the vector $\mathbf{p}_{i}=\left(x_{i}, y_{i}, z_{i}\right)^{T}$ represent the position of the $i$ th particle in a rectangular inertial reference frame [8]. We assume that the $i$ th particle is subjected to the given impressed force $\mathbf{F}_{i}(t)$, so that its acceleration without constraints would be given by the vector $\mathbf{a}_{i}=\mathbf{F}_{i}(t) / m_{i}$. The three components of the vector $\mathbf{a}_{i}$ correspond to the accelerations of the $i$ th particle driven by $\mathbf{F}_{i}$ in the three mutually perpendicular coordinate directions. Thus the equation of motion without constraints on the particles of the system, can be written as

$$
\mathbf{M a}(t)=\mathbf{F}(\mathbf{x}(t), \dot{\mathbf{x}}(t), t),
$$

where

$$
\begin{aligned}
\mathbf{F}(t) & =\left(\mathbf{F}_{1}^{T}, \mathbf{F}_{2}^{T}, \ldots, \mathbf{F}_{n}^{T}\right)^{T} \\
\mathbf{a}(t) & =\left(\mathbf{a}_{1}^{T}, \mathbf{a}_{2}^{T}, \ldots, \mathbf{a}_{n}^{T}\right)^{T} \\
\mathbf{x}(t) & =\left(\mathbf{p}_{1}^{T}, \mathbf{p}_{2}^{T}, \ldots, \mathbf{p}_{n}^{T}\right)^{T} \\
\mathbf{M} & =\operatorname{Diag}\left(m_{1}, m_{1}, m_{1}, m_{2}, \ldots, m_{n}, m_{n}, m_{n}\right) .
\end{aligned}
$$

In the presence of constraints, the acceleration of the particles at time $t$ will differ from $\mathbf{a}(t)$ and we donate this acceleration by the $3 n$-vector $\ddot{\mathbf{x}}(t)=\left(\ddot{\mathbf{p}}_{1}^{T}, \ddot{\mathbf{p}}_{2}^{T}, \ldots, \ddot{\mathbf{p}}_{n}^{T}\right)^{T}$. Gauss's principle asserts that among all the accelerations that the system can have at time $t$ which are compatible with the constraints, the accelerations that actually materialize are those that minimize

$$
\begin{aligned}
G(\ddot{\mathbf{x}}) & =(\ddot{\mathbf{x}}-\mathbf{a})^{T} \mathbf{M}(\ddot{\mathbf{x}}-\mathbf{a}) \\
& =\left(\mathbf{M}^{1 / 2} \ddot{\mathbf{x}}-\mathbf{M}^{1 / 2} \mathbf{a}\right)^{T}\left(\mathbf{M}^{1 / 2} \ddot{\mathbf{x}}-\mathbf{M}^{1 / 2} \mathbf{a}\right) .
\end{aligned}
$$

Assume that the $m$ constraints can be expressed as linear equality relations between the accelerations of the particles of the system, then the constraints will always be of the standard form

$$
\mathbf{A}(\dot{\mathbf{x}}, \mathbf{x}, t) \ddot{\mathbf{x}}=\mathbf{b}(\dot{\mathbf{x}}, \mathbf{x}, t)
$$

where the matrix $\mathbf{A}$ is $m$ by $3 n$ and the vector $\mathbf{b}$ is an $m$ vector.

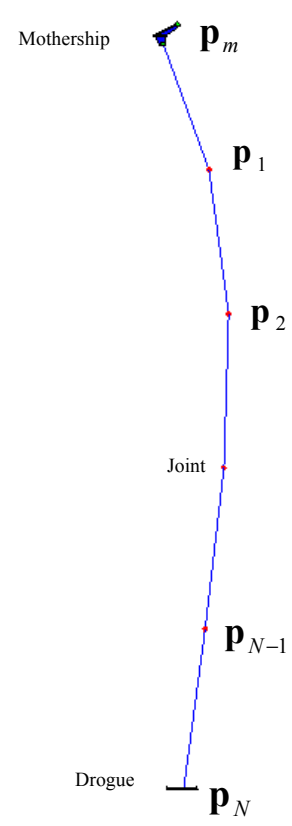

Fig. 2. Cable-drogue systems based on lumped mass representation

Minimizing (2) subject to the constraint (3) implies that at each instant of time $t$, the actual acceleration of the system 
of $n$ particles is given by

$$
\ddot{\mathbf{x}}=\mathbf{a}+\mathbf{M}^{-1 / 2}\left(\mathbf{A} \mathbf{M}^{-1 / 2}\right)^{+}(\mathbf{b}-\mathbf{A} \mathbf{a}),
$$

where $(\cdot)^{+}$is the unique Moore-Penrose inverse [8].

\section{B. Dynamics Equations of Cable-Drogue Systems}

Figure 2 depicts the cable-drogue systems with an Nlink cable modelled as a finite number of rigid links. The forces acting on each link are lumped and applied at the joint. The drogue is the last joint of the cable. Let $\mathbf{p}_{i}=$ $\left(x_{i}, y_{i}, z_{i}\right)^{T} \in \mathbf{R}^{3}, i=1,2, \ldots, N$ be the location of the $i$ th link. The position of the towplane or mothership is $\mathbf{p}_{m}=$ $\left(x_{m}, y_{m}, z_{m}\right)^{T} \in \mathbf{R}^{3}$. If the point masses associated with each link are unconstrained, then the dynamics equations describing their motions are

$$
\begin{aligned}
\ddot{\mathbf{p}}_{i} & =\mathbf{a}_{i}, \quad i=1,2, \ldots, N, \\
\ddot{\mathbf{p}}_{m} & =\mathbf{a}_{m},
\end{aligned}
$$

where $\mathbf{a}_{i}, i=1,2, \ldots, N$, and $\mathbf{a}_{m} \in \mathbf{R}^{3}$ are the unconstrained accelerations driven by the applied forces in three dimensions. Alternatively, defining $\mathbf{x}=\left(\mathbf{p}_{1}^{T}, \mathbf{p}_{2}^{T}, \cdots, \mathbf{p}_{N}^{T}\right)^{T}$ and $\mathbf{a}=\left(\mathbf{a}_{1}^{T}, \mathbf{a}_{2}^{T}, \cdots, \mathbf{a}_{N}^{T}\right)^{T}$ gives

$$
\ddot{\mathbf{x}}=\mathbf{a} .
$$

However, the motions of the point masses associated with the link are constrained by the relationship

$$
\begin{aligned}
\left\|\mathbf{p}_{1}-\mathbf{p}_{m}\right\|^{2} & =l^{2}, \\
\left\|\mathbf{p}_{i+1}-\mathbf{p}_{i}\right\|^{2} & =l^{2}, i=1,2, \ldots, N-1, \\
l & =L / N,
\end{aligned}
$$

where $L$ is the cable length, or alternatively as the position constraint

$$
\phi\left(\mathbf{x} ; \mathbf{p}_{m}\right) \triangleq\left(\begin{array}{c}
\left\|\mathbf{p}_{1}-\mathbf{p}_{m}\right\|^{2}-l^{2} \\
\left\|\mathbf{p}_{2}-\mathbf{p}_{1}\right\|^{2}-l^{2} \\
\vdots \\
\left\|\mathbf{p}_{N}-\mathbf{p}_{N-1}\right\|^{2}-l^{2}
\end{array}\right)=0
$$

Differentiating Equation (6) with respect to time results in the velocity constraint

$$
\psi\left(\mathbf{x} ; \mathbf{p}_{m}\right) \triangleq\left(\begin{array}{c}
\left(\mathbf{p}_{1}-\mathbf{p}_{m}\right)^{T}\left(\dot{\mathbf{p}}_{1}-\dot{\mathbf{p}}_{m}\right) \\
\left(\mathbf{p}_{2}-\mathbf{p}_{1}\right)^{T}\left(\dot{\mathbf{p}}_{2}-\dot{\mathbf{p}}_{1}\right) \\
\vdots \\
\left(\mathbf{p}_{N}-\mathbf{p}_{N-1}\right)^{T}\left(\dot{\mathbf{p}}_{N}-\dot{\mathbf{p}}_{N-1}\right)
\end{array}\right)=0 .
$$

Differentiating Equation (7) results in the acceleration constraint

$$
\left(\begin{array}{c}
\left(\mathbf{p}_{1}-\mathbf{p}_{m}\right)^{T}\left(\ddot{\mathbf{p}}_{1}-\ddot{\mathbf{p}}_{m}\right)+\left\|\dot{\mathbf{p}}_{1}-\dot{\mathbf{p}}_{m}\right\|^{2} \\
\left(\mathbf{p}_{2}-\mathbf{p}_{1}\right)^{T}\left(\ddot{\mathbf{p}}_{2}-\ddot{\mathbf{p}}_{1}\right)+\left\|\dot{\mathbf{p}}_{2}-\dot{\mathbf{p}}_{1}\right\|^{2} \\
\vdots \\
\left(\mathbf{p}_{N}-\mathbf{p}_{N-1}\right)^{T}\left(\ddot{\mathbf{p}}_{N}-\ddot{\mathbf{p}}_{N-1}\right)+\left\|\dot{\mathbf{p}}_{N}-\dot{\mathbf{p}}_{N-1}\right\|^{2}
\end{array}\right)=0
$$

which, assuming the motion of the mothership is known, can be written in matrix notation as

$$
\mathbf{A}(\mathbf{x}) \ddot{\mathbf{x}}=\mathbf{b}\left(\dot{\mathbf{x}}, \dot{\mathbf{p}}_{m}, \ddot{\mathbf{p}}_{m}\right)
$$

where

$$
\begin{aligned}
& \mathbf{A}=\left(\begin{array}{cc}
\left(\mathbf{p}_{1}-\mathbf{p}_{m}\right)^{T} & 0 \\
-\left(\mathbf{p}_{2}-\mathbf{p}_{1}\right)^{T} & \left(\mathbf{p}_{2}-\mathbf{p}_{1}\right)^{T} \\
\vdots & \ddots \\
0 & \cdots
\end{array}\right. \\
& \cdots \quad 0 \\
& \left.\begin{array}{cc}
\vdots & \ddots \\
-\left(\mathbf{p}_{N}-\mathbf{p}_{N-1}\right)^{T} & \left(\mathbf{p}_{N}-\mathbf{p}_{N-1}\right)^{T}
\end{array}\right) \\
& \mathbf{b}=-\left(\begin{array}{c}
\left\|\dot{\mathbf{p}}_{1}-\dot{\mathbf{p}}_{m}\right\|^{2} \\
\left\|\dot{\mathbf{p}}_{2}-\dot{\mathbf{p}}_{1}\right\|^{2} \\
\vdots \\
\left\|\dot{\mathbf{p}}_{N}-\dot{\mathbf{p}}_{N-1}\right\|^{2}
\end{array}\right)+\left(\begin{array}{c}
\left(\mathbf{p}_{1}-\mathbf{p}_{m}\right)^{T} \ddot{\mathbf{p}}_{m} \\
0 \\
\vdots \\
0
\end{array}\right) .
\end{aligned}
$$

The mass matrix $\mathbf{M}=\operatorname{Diag}\left(m_{l}, m_{l}, \ldots, m_{l}, m_{d}, m_{d}, m_{d}\right)$ $\in \mathbf{R}^{3 N \times 3 N}$, where $m_{l}=m_{c} / N$ is the mass of each link, $m_{c}$ is the mass of the cable, and $m_{d}$ is the mass of the drogue.

Therefore, based on Gauss's principle, the actual acceleration of the cable-drogue system (5) subject to the constraints (8) is given by Equation (4). The initial conditions for the system must be chosen such that both $\phi\left(\mathbf{x} ; \mathbf{p}_{m}\right)=0$ and $\psi\left(\mathbf{x} ; \mathbf{p}_{m}\right)=0$.

However, as indicated by [9], one of the drawbacks of this method is that while solving Equation (4), numerical error may cause the constraints $\phi\left(\mathbf{x} ; \mathbf{p}_{m}\right)$ and $\psi\left(\mathbf{x} ; \mathbf{p}_{m}\right)$ to drift from zero. When this happens, Equation (4) no longer represents the physical dynamics of the cable. That is to say, no mechanism serves to drive the constraints back to zero.

To solve this problem, Equation (4) is modified as [9]

$$
\begin{aligned}
\ddot{\mathbf{x}}=\mathbf{a} & +\mathbf{M}^{-1 / 2}\left(\mathbf{A} \mathbf{M}^{-1 / 2}\right)^{+}(\mathbf{b}-\mathbf{A a}) \\
& -\gamma_{1}\left(\frac{\partial \phi}{\partial \mathbf{x}}\right)^{T} \phi-\gamma_{2}\left(\frac{\partial \psi}{\partial \mathbf{x}}\right)^{T} \psi,
\end{aligned}
$$

where $\gamma_{1}$ and $\gamma_{2}$ are positive constants.

The additional two terms cause the ODE solution to decrease the gradient of the constraints until they are not violated. Selecting $\gamma_{1}$ and $\gamma_{2}$ properly guarantees that the modified equation approximately represents the dynamics of the constrained physical system.

\section{Force Equations of Cable-Drogue Systems}

1) Applied Forces on the Cable: The applied forces which causes the unconstrained acceleration on the joints consist of gravity and aerodynamic forces.

(1) The gravity term has the form

$$
\mathbf{F}_{g}=m g \mathbf{e}_{3},
$$

where $\mathbf{e}_{3}=(0,0,1)^{T}$.

(2) The aerodynamic forces acting on the cable can be determined based on the crossflow principle in [3] and [6]. The drag and lift coefficients for an inclined cylinder are 
given by

$$
\begin{aligned}
C_{D_{j}} & =C_{f_{j}}+C_{n_{j}} \sin ^{3}\left(\alpha_{j}\right), \\
C_{L_{j}} & =C_{n_{j}} \sin ^{2}\left(\alpha_{j}\right) \cos \left(\alpha_{j}\right), \\
& j=1,2, \cdots, N-1,
\end{aligned}
$$

where $\alpha_{j}$ is the angle of attack of the $j$ th segment, and

$$
\begin{gathered}
C_{f_{j}}= \begin{cases}0.038-0.0425 M_{p_{j}}, & M_{p_{j}}<0.4 \\
0.013+0.0395\left(M_{p_{j}}-0.85\right)^{2}, & M_{p_{j}} \geq 0.4\end{cases} \\
C_{n_{j}}=1.17+M_{n_{j}} / 40-M_{n_{j}}^{2} / 4+5 M_{n_{j}}^{3} / 8, \\
j=1,2, \cdots, N-1,
\end{gathered}
$$

where $M_{p_{j}}$ is the Mach number parallel to the cable segment, and $M_{n_{j}}$ is the Mach number normal to the cable segment [6] [3]. The angle of attack of the $j$ th segment $\alpha_{j}$ can be computed as

$$
\cos \alpha_{j}=\frac{\mathbf{l}_{j} \cdot, \mathbf{v}_{j}}{\left\|\mathbf{l}_{j}\right\|\left\|\mathbf{v}_{j}\right\|}, j=1,2, \cdots, N-1,
$$

where $\mathbf{l}_{j}=\mathbf{p}_{j-1}-\mathbf{p}_{j}$ and $\mathbf{v}_{j}$ is the velocity of the $j$ th segment. The unit vectors defining the directions of the drag and lift vectors in the inertia frame are

$$
\begin{gathered}
\mathbf{e}_{D_{j}}=-\frac{\mathbf{v}_{j}}{\left\|\mathbf{v}_{j}\right\|}, \\
\mathbf{e}_{L_{j}}=-\frac{\left(\mathbf{v}_{j} \times \mathbf{l}_{j}\right) \times \mathbf{v}_{j}}{\left\|\left(\mathbf{v}_{j} \times \mathbf{l}_{j}\right) \times \mathbf{v}_{j}\right\|}, \\
j=1,2, \cdots, N-1,
\end{gathered}
$$

Hence the drag and lift forces vectors are

$$
\begin{aligned}
\mathbf{F}_{j}^{\text {drag }} & =\frac{1}{2} \rho C_{D_{j}} l_{j} d\left\|\mathbf{v}_{j}\right\|^{2} \mathbf{e}_{D_{j}}, \\
\mathbf{F}_{j}^{l i f t} & =\frac{1}{2} \rho C_{L_{j}} l_{j} d\left\|\mathbf{v}_{j}\right\|^{2} \mathbf{e}_{L_{j}}, \\
j & =1,2, \cdots, N-1,
\end{aligned}
$$

where $\rho$ is the atmospheric density, $l_{j}$ is the length of the $j$ th cable segment, and $d$ is the diameter of the cable segment.

2) Applied Forces on the drogue: To facilitate rendezvous with the MAV, the drogue is designed as a UAV-like aircraft with autopilot, communication system, and a catch mechanism. The applied forces on the drogue are aerodynamic drag and lift, which can be written as

$$
\begin{gathered}
\mathbf{F}_{N}^{\text {drag }}=-\frac{1}{2} \rho C_{D_{N}} S\left\|\mathbf{v}_{N}\right\| \mathbf{v}_{N}, \\
\mathbf{F}_{N}^{\text {lift }}=\frac{1}{2} \rho C_{L_{N}} S\left\|\mathbf{v}_{N}\right\|^{2} \mathbf{e}_{L_{N}},
\end{gathered}
$$

where $S$ is the incidence area of the drogue, $\mathbf{v}_{N}$ is the velocity vector of the drogue, $\mathbf{e}_{L_{N}}$ is the unit vector defining the lift force direction of the drogue in the inertia frame and $C_{D_{N}}$ and $C_{L_{N}}$ are drag and lift coefficients respectively.

\section{Drogue Control}

If the towplane is in a constant-angular-rate orbit of radius $\mathrm{R}$, and the drogue has sufficient aerodynamic drag, then the motion of the drogue has a stable orbit of radius $r \ll R$. Since the angular rates of the towplane and the drogue are identical, i.e.

$$
\omega=\frac{V_{m}}{R}=\frac{V_{N}}{r},
$$

where $\omega$ is the angular rate of the towplane and the drogue, and the $V_{m}$ and $V_{N}$ are the airspeeds of the mothership and the drogue respectively, then

$$
V_{N}=r \frac{V_{m}}{R}
$$

Therefore, we can regulate the drogue to a desired radius $r^{d}$, by regulating the velocity of the drogue to $V_{N}^{d}=r^{d} V_{m} / R$.

\section{A. Utilization of the aerodynamics drag coefficient}

A possible strategy for controlling the velocity of the drogue is to change its drag coefficient using control surfaces. By analyzing the relationship between drogue radius and drag coefficient, we found that the larger drag coefficient corresponds to the smaller radius of the drogue.

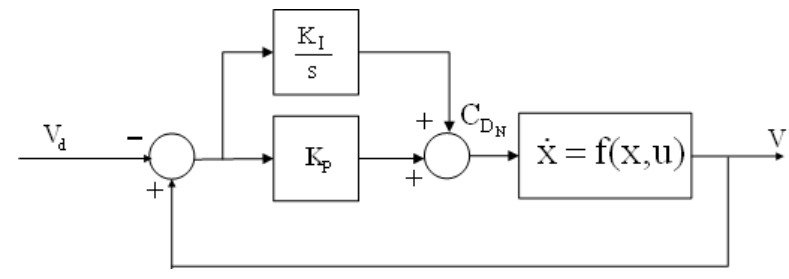

Fig. 3. The layout of the PI controller based on drag coefficient.

As shown in Figure 3, a possible control law of the drag coefficient is

$$
C_{D_{N}}=\left(K_{P}+\frac{K_{I}}{s}\right)\left(V_{N}-V_{N}^{d}\right)
$$

where $V_{N}{ }^{d}$ is the desired airspeed of the drogue, the $V_{N}$ is the current airspeed, and $K_{P}$ and $K_{I}$ are positive proportional and integral gains repectively.

\section{Simulation Results}

This section describes several simulations that illustrate the application of Gauss's Principle in constructing the cabledrogue dynamics and the controllability of the drogue by using the aerodynamic drag coefficient.

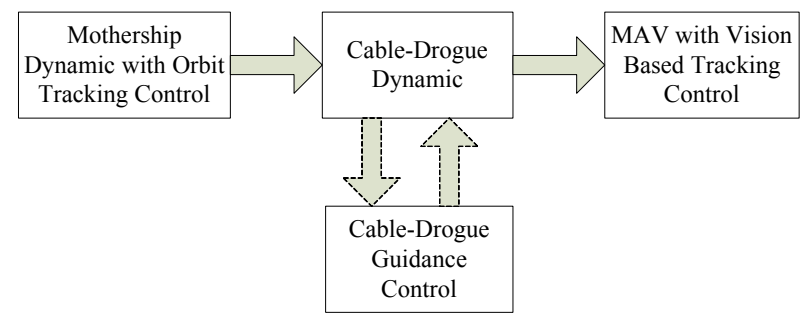

Fig. 4. This figure shows the structure of the simulation system. The mothership flies in a constant-radius orbit and does not feel tension from cable-drogue system. We can choose to control the drogue or not. A visionbased algorithm is implemented on the MAV to track the drogue. 
The simulation architecture is shown as Figure 4. To simplify the problem, wind is not considered in this paper, and the air density does not vary as a function of altitude. The parameters used for the simulations are shown in Table I

TABLE I

SiMUlation PARAMETERS

\begin{tabular}{ccccc}
\hline \hline Mothership & $\begin{array}{c}\text { Airspeed } \\
50 \mathrm{~m} / \mathrm{s}\end{array}$ & $\begin{array}{c}\text { Altitude } \\
1000 \mathrm{~m}\end{array}$ & $\begin{array}{c}\text { Orbit Radius } \\
300 \mathrm{~m}\end{array}$ \\
\hline \multirow{2}{*}{ Cable } & Links & Length & Diameter & Mass \\
& 10 & $900 \mathrm{~m}$ & $0.01 \mathrm{~m}$ & $1 \mathrm{~kg}$ \\
\hline \multirow{2}{*}{ MAV } & Airspeed & & & \\
& $16.67 \mathrm{~m} / \mathrm{s}$ & & \\
\hline \hline
\end{tabular}

\section{A. Circular motion of the system without drogue control}

In the first scenario the drogue is not controlled. Figure 5 shows the North-East motion of the drogue, and we can see that the drogue eventually enters a steady orbit. Figure 6 shows the steady drogue radius oscillates between $115 \mathrm{~m}$ and $119 \mathrm{~m}$. The steady state drogue velocity is approximately $19.5 \mathrm{~m} / \mathrm{s}$ which is larger than the velocity of the MAV, which will prevent recovery. Figure 7 and Figure 8 show the 2D top-down view and 3D view of the simulated system. The cable bends outward under the effect of the aerodynamic drag.

B. Circular motion of the system under the drag coefficient control

The second simulation was performed to demonstrate that the drag coefficient can be effectively used to control the drogue radius. In order to compare the difference between the system motion with and without control, we apply the actuation signal after 150 seconds which is the time that the drogue enters steady state.

If the desired drogue radius is $60 \mathrm{~m}$, then the desired drogue velocity is $V_{N}^{d}=10 \mathrm{~m} / \mathrm{s}$.

Figure 9 shows that the steady state radius of the drogue converges to a smaller radius. Figure 10 shows that drag based control approach works well and after 250 seconds the three items enter steady states. The steady state radius oscillates between $54 \mathrm{~m}$ and $58 \mathrm{~m}$. The steady state velocity oscillates between $9 \mathrm{~m} / \mathrm{s}$ and $9.5 \mathrm{~m} / \mathrm{s}$ allowing potential aerial recovery.

\section{CONCLUSION}

A novel approach to Aerial Recovery of Micro Air Vehicles has been presented. The multi-link cable-drogue dynamic model is established based on Gauss's Principle which provides a succinct method to model the system with complex constraints. The control strategy using drag coefficient is effective in controlling the simulation. Two simulations have been developed and used to simulate the motion of the aerial recovery systems. The simulation results illustrate the feasibility of the concept.
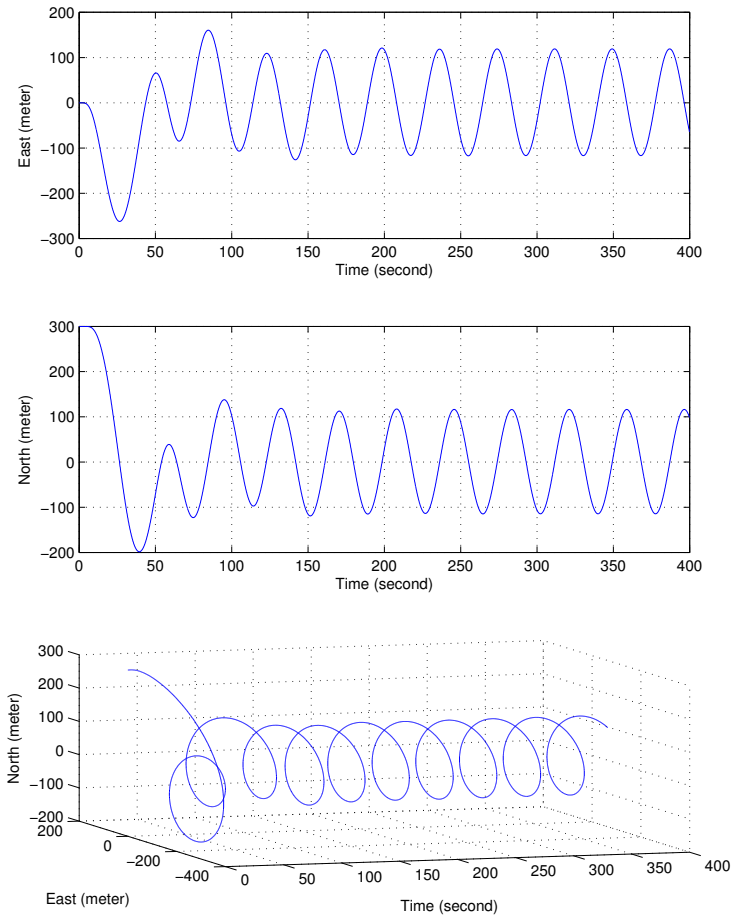

Fig. 5. North-East motion of the drogue.

\section{ACKNOWLEDGMENT}

This research was supported by the Air Force Office of Scientific Research under STTR contract No. FA 955009-C-0102 to Procerus Technologies and Brigham Young University.

\section{REFERENCES}

[1] R. A. Skop and Y. Choo, "The configuration of a cable towed in a circular path," Journal of Aircraft, vol. 8, pp. 856-862, November 1971.

[2] R. Murray, "Trajectory generation for a towed cable system using differential flatness," 13th Triennial World Congress of the International Federation of Automatic Control, pp. 395-400, 1996.

[3] T. P. Williams, P., "Dynamics of circularly towed cable systems, part 1: Optimal configurations and their stability," AIAA Journal of Guidance Control and Dynamics, vol. 30, pp. 753-765, May-June 2007.

[4] T. P. Williams, P., "Dynamics of circularly towed cable systems, part 2: Transitional flight and deployment control," AIAA Journal of Guidance Control and Dynamics, vol. 30, pp. 766-779, May-June 2007.

[5] M. R. Chin, C.K.H. and H. Connell, "A numerical model of a towed cable-body system," ANZIAM Journal, vol. 42, pp. C362-C384, 2000.

[6] T. S. N. J. E. Cochran Jr., M. Innocent and A. Thukral, "Dynamics and control of maneuverable towed flight vehicles," AIAA Journal of Guidance, Control and Dynamics, vol. 15, no. 5, pp. 1245-1252, 1992.

[7] Y. Turkyilmaz and O. Egeland, "Active depth control of towed cables in 2d," IEEE Conference on Decision and Control, pp. 952-957, December 2001.

[8] F. E. Udwadia and R. E. Kalaba, Analytical Dynamics: A New Approach. Cambridge University Press, 1996.

[9] T. W. McLain and R. W. Beard, "Trajectory planning for coordinated rendezvous of unmanned air vehicles," vol. 4369, pp. 1-8, 2000. 

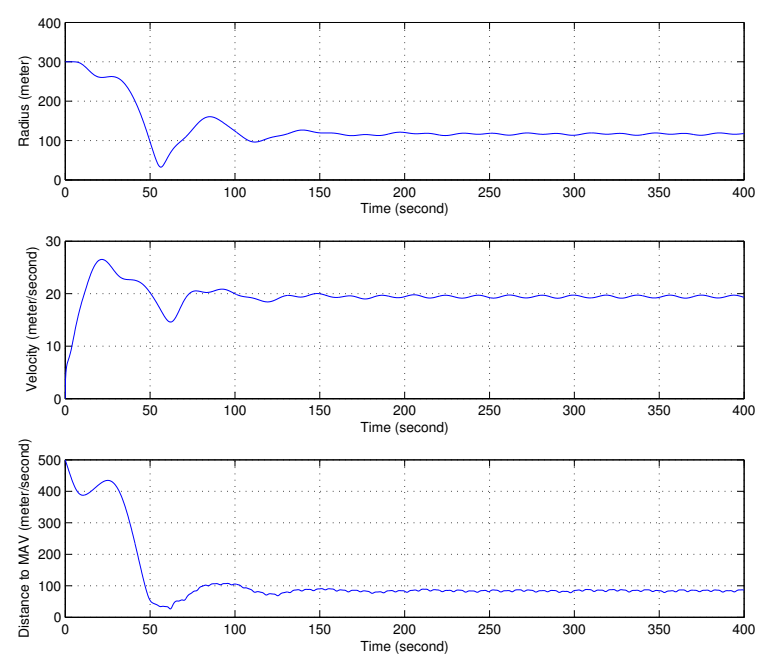

Fig. 6. This figure shows the radius, velocity and distance to the MAV of the drogue responses. After 150 seconds, the three items enter steady states.

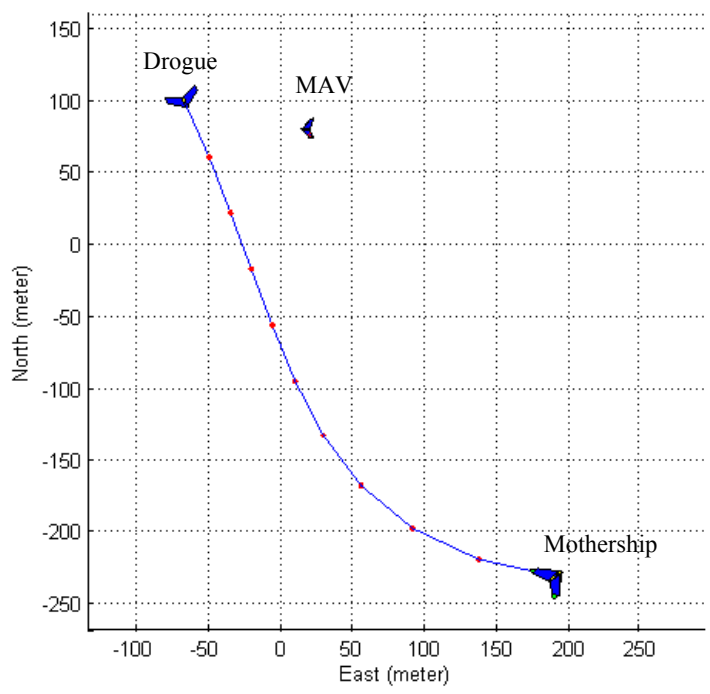

Fig. 7. Top-down view of the simulated system. The dots on the cable are the joints.

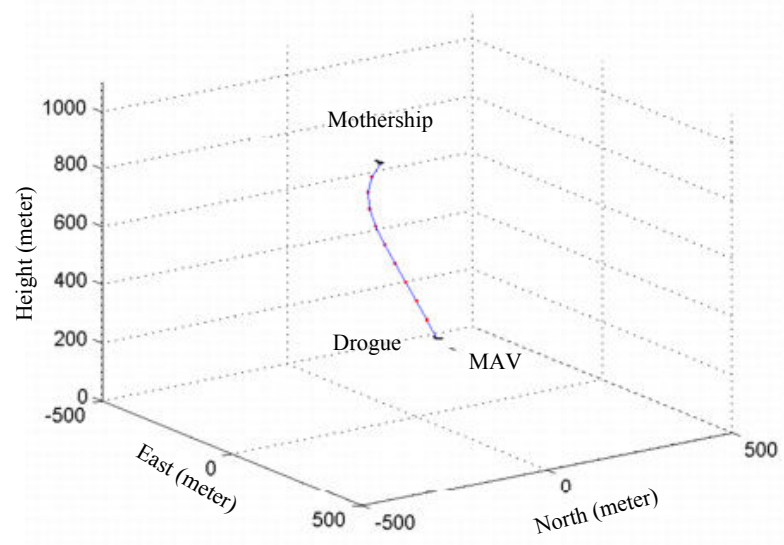

Fig. 8. 3D view of the simulated system.
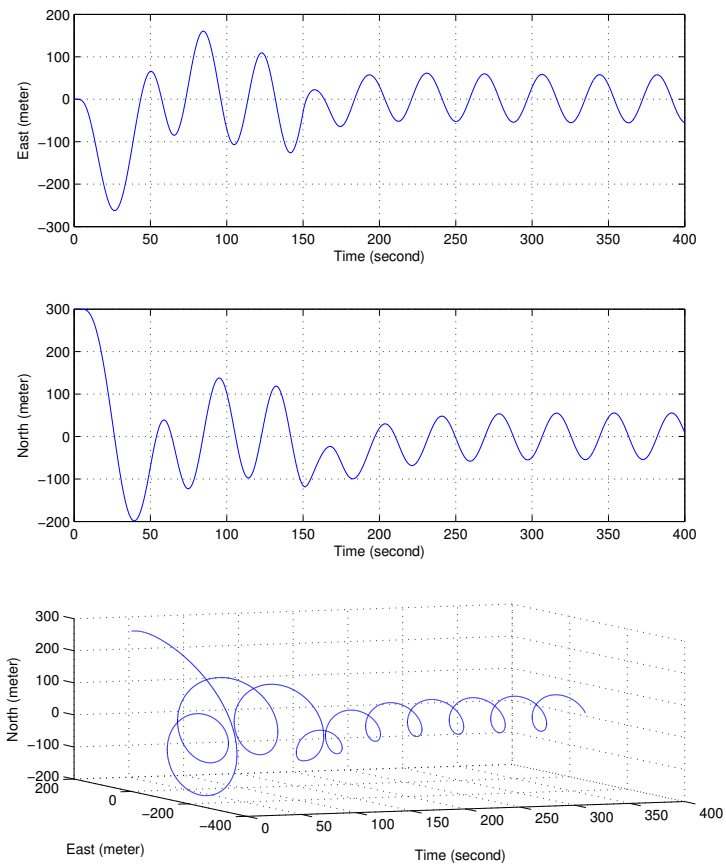

Fig. 9. North-East motion of the drogue under control. The steady state radius is smaller than that without control.
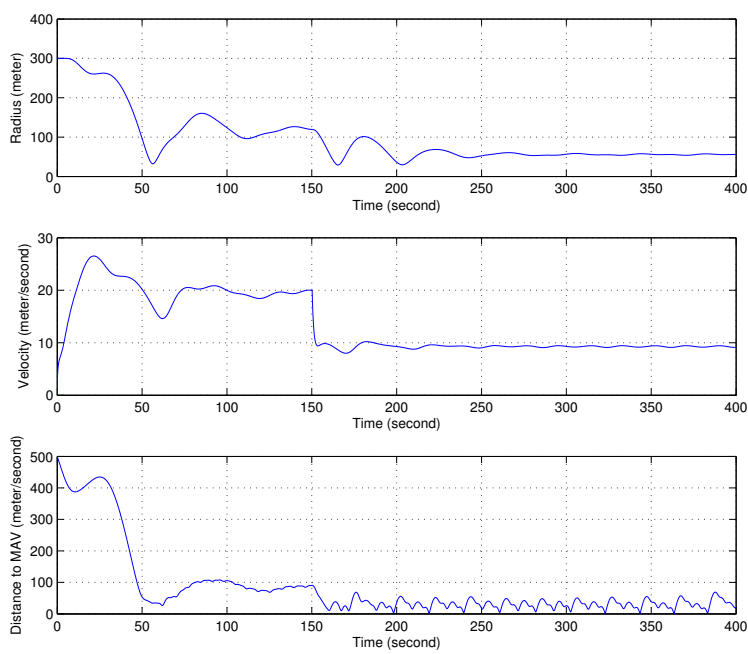

Fig. 10. This figure shows the radius, velocity and distance to the MAV of the drogue responses under drag coefficient control. After 250 seconds, the three items enter steady states. 